\title{
NOUVELLE
}

\section{Le mystère de la disparition des histones au cours de la spermatogenèse}

Sophie Barral, Yuichi Morozumi, Naghmeh Hoghoughi, Sophie Rousseaux, Saadi Khochbin
CNRS UMR 5309 ; Inserm, U1209 ; université Grenoble Alpes ; Institute for Advanced Biosciences, Grenoble, F-38700 France. saadi.khochbin@univ-grenoble-alpes.fr
L'empaquetage du génome mâle, un processus qui reste mystérieux Le spermatozoïde est la seule cellule capable de survivre en dehors de l'organisme producteur. Il s'agit d'une cellule hautement spécialisée qui se prépare à quitter l'organe génital mâle et à survivre dans les voies génitales femelles, lieu de la fécondation. La mission du spermatozoïde est de transporter une copie du génome mâle jusqu'à l'ovocyte pour le livrer lors de la fusion gamétique. Le processus qui aboutit à la production des spermatozoïdes est connu sous le nom de spermatogenèse. La différenciation des cellules germinales mâles progénitrices comprend deux étapes principales. La première implique la méiose au cours de laquelle le génome est initialement brassé puis divisé, aboutissant à la production de quatre cellules nommées spermatides, portant chacune une seule copie du génome. Au cours de la deuxième étape, ou spermiogénèse, les gamètes mâles se préparent pour leur futur voyage à l'extérieur de l'organisme de production vers le lieu de fécondation. Lors de cette étape post-méiotique, les gamètes mâles sont le siège d'une véritable métamorphose à l'issue de laquelle ces cellules seront dotées d'un flagelle, indispensable pour la mobilité des futurs spermatozoïdes. Concomitamment, ils subissent une réorganisation spectaculaire et unique de leur génome. Le fait marquant de cette différenciation post-méiotique est la disparition des nucléosomes ${ }^{1}$, unités universelles d'organisation et d'empaquetage des génomes chez les eucaryotes.

La disparition des histones, les constituants des nucléosomes, au cours de la spermiogenèse et leur remplacement par des petites protéines basiques nonhistones, les protéines de transition et les protamines ont été précédemment décrits [1]. Cette transformation globale de l'organisation du génome dans les cellules germinales mâles a comme objectif la compaction extrême de celuici avant le voyage du spermatozoïde afin d'assurer sa protection lors de cette période à risque pour son intégrité.

De nombreux travaux ont désormais montré que toute perturbation affectant le remplacement des histones aboutissait à une compaction défectueuse du génome dans le spermatozoïde et à une infertilité masculine. Le remplacement des histones est donc une étape critique pour la procréation et la perpétuation de l'espèce. Néanmoins, une question fondamentale reste ouverte: nous ne savons pas comment les histones sont dissociées de l'ADN et disparaissent, ni comment cette dissociation des histones est couplée à l'assemblage des protéines non-histones et à l'empaquetage du génome. En d'autres termes, I'ADN se structure autour des protamines et non plus des histones, mais nous ne compre-

${ }^{1}$ Le nucléosome est composé d'un fragment d'ADN (environ 147 paires de bases) enroulé autour d'un octamère d'histones. nons pas comment les nucléosomes sont remplacés par les nucléoprotamines.

La vision classique et largement documentée est que les histones sont initialement remplacées par des protéines dites «de transition » elles-mêmes substituées, à leur tour, par les protamines.

Malgré l'importance de ces questions, nous ne disposons d'aucune information quant à la compréhension des mécanismes qui régissent ces bouleversements dans l'organisation du génome. Or la connaissance des mécanismes sousjacents est susceptible d'influencer non seulement la biologie de la chromatine et l'épigénétique, mais aussi de nous éclairer sur les éléments déterminants d'une fertilité masculine optimale.

Le remplacement des histones : par qui et comment?

Malgré l'identification et la caractérisation des protéines non-histones qui remplacent les histones au cours de la spermiogénèse, la relation fonctionnelle entre histones, protéines de transition et protamines est longtemps restée obscure. L'observation de l'apparition successive des protéines de transition puis des protamines avait initialement suggéré une action séquentielle de cellesci dans la réorganisation du génome. Lors d'une première étape, les histones étaient remplacées par les protéines de transition et dans une deuxième phase, les protamines chassaient les protéines de transition et dirigeaient la compaction finale du génome $[2,3]$. Cependant, des observations ont semé le doute sur 


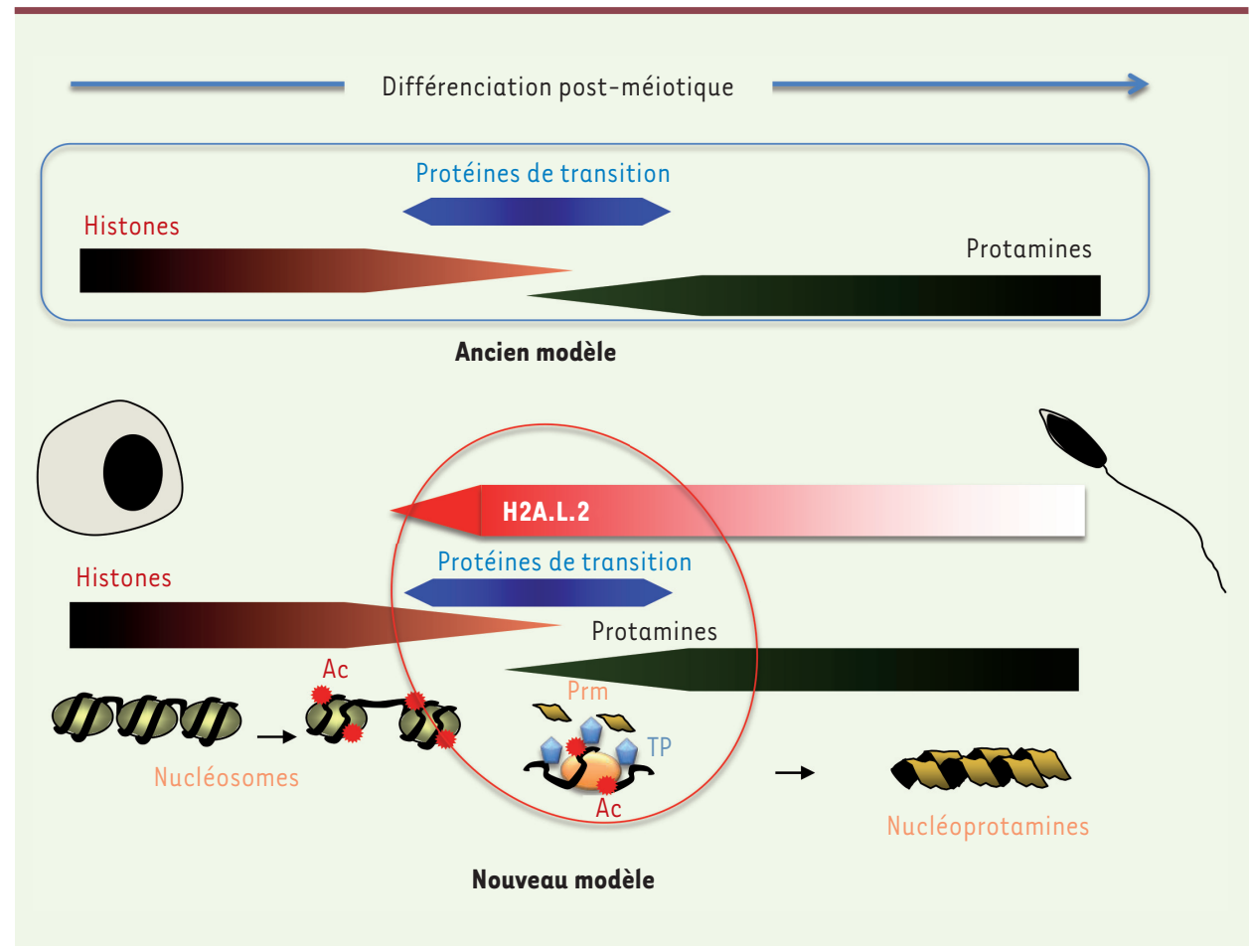

Figure 1. Nouveau modèle résumant les étapes impliquées dans le remplacement des histones au cours de la spermatogenèse. Le schéma général du remplacement des histones largement répandu proposait l'action, par étapes successives et distinctes, des protéines de transition (TP) remplaçant les histones, puis des protamines (Prm) se substituant aux protéines de transition (modèle ancien, partie supérieure). Nos travaux [8] mettent en évidence une étape supplémentaire qui correspond à la synthèse concomitante du variant d'histone H2A.L.2 et des protéines de transition. Leur action simultanée facilite l'incorporation des protamines. Ce nouveau modèle est présenté dans la partie inférieure de la figure. Ac: acétylation.

cette hypothèse du remplacement des histones en deux étapes successives, l'ordre d'apparition des protéines remplaçant les histones ne semblant pas aussi clair qu'initialement pensé [3]. $\varepsilon$ n effet, les protéines de transition, les protamines et les histones peuvent être simultanément détectées dans la même cellule [4]. D’autres expériences, notamment la génération de souris déficientes (double Knock Out) pour les deux gènes codant les protéines de transition, ont rendu la situation encore plus confuse. Ces travaux ont montré qu'en l'absence totale des protéines de transition, le remplacement des histones se produisait [4-6]. Cette dernière observation pose donc la question essentielle du rôle des protéines de transition dans le processus de remplacement des histones. Quel est leur rôle? Les expériences de double extinction des gènes les codant ont montré que ces protéines sont nécessaires pour une compaction efficace du génome par les protamines ainsi que pour la maturation de la protamine 2 (Prm2). La protamine 2 est synthétisée sous la forme d'une pré-protéine qui subit un clivage pro- téolytique dans sa partie $\mathrm{N}$-terminale lors de son assemblage. L'accumulation du précurseur pré-Prm2 en l'absence des protéines de transition indique un rôle pour ces protéines dans le contrôle de l'assemblage des protamines. Cependant, aucune donnée publiée ne laissait entrevoir quelle pouvait être la relation fonctionnelle entre les protéines de transition et les protamines.

L'invasion du nucléosome : une histone jouant le rôle de « cheval de Troie » La découverte en 2007 [7], d'une série de variants d'histones de type $\mathrm{H} 2 \mathrm{~B}$ et H2A exprimés spécifiquement dans les cellules spermatogéniques a permis de reconsidérer le remplacement des histones sous un nouveau jour. En effet, parmi ces histones, un variant de H2A nommé H2A.L.2 est exprimé tardivement, pratiquement en même temps que les protéines de transition, et de surcroît dans les mêmes cellules que celles-ci [8]. Ces observations ont désigné H2A.L.2 comme un facteur particulièrement intéressant, car il s'agit d'une histone synthétisée au moment même du remplacement général des histones.
Cette situation inattendue suggérait que cette histone pouvait jouer un rôle dans la transformation des nucléosomes et plus précisément, qu'elle pouvait être un nouvel acteur dans le jeu d'interactions entre les protéines de transition et les protamines. L'observation de la spermatogenèse des souris invalidées pour le gène codant H2A.L.2 a considérablement renforcé cette hypothèse. Ces souris ont en effet exactement le même phénotype que celles doublement déficientes pour les gènes codant les protéines de transition, à savoir une perturbation de la maturation de la protéine pré-Prm2, une compaction défectueuse du génome dans les spermatozoïdes et une infertilité des mâles. Ces observations suggèrent qu'en l'absence de l'histone H2A.L.2, malgré une synthèse normale, les protéines de transition restent non-fonctionnelles. Ainsi, l'absence du variant H2A.L.2 serait donc fonctionnellement équivalente à la carence en protéines de transition. Autrement dit, l'histone H2A.L.2 serait nécessaire aux protéines de transition pour assurer leur fonction.

Une série d'expériences visant à vérifier cette hypothèse a mis en évidence le 
mécanisme par lequel l'histone H2A.L.2 facilite l'action des protéines de transition: l'incorporation de l'histone H2A.L.2 ouvre le nucléosome, ce qui permet aux protéines de transition d'être chargées sur la chromatine et d'interagir avec les protamines tout en assurant la maturation du précurseur pré-Prm2. En l'absence de H2A.L.2, les protéines de transition ne peuvent pas entrer efficacement en interaction avec la chromatine et s'agrègent donc en piégeant une fraction des protamines qui ne peuvent pas être maturées ni assemblées. La conséquence de cette situation est un sous-dosage de protamines au niveau de l'ADN et une compaction défectueuse du génome [8]. L’histone H2A.L.2 se comporte donc comme un cheval de Troie : une fois incorporée au sein du nucléosome, ce variant d'histone ouvre la structure et permet l'invasion du nucléosome par des protéines non-histones.

\section{Conclusion}

L'étude fonctionnelle de l'histone H2A.L.2 a permis de mieux comprendre le phénomène mystérieux de la disparition à grande échelle des histones, qui survient au cours de la phase postméiotique de la spermatogenèse. La stratégie utilisée par la nature est de favoriser un changement structural du nucléosome et une déstabilisation de celui-ci à l'échelle du génome par la synthèse d'un variant d'histone de type H2A produit en même temps que les protéines spécialisées qui remplacent les histones. Nous savons néanmoins que cette étape à elle seule n'est pas suffisante pour assurer cette transformation du nucléosome. Le remplacement se produit dans un contexte où le nucléosome doit préalablement être hyper-acétylé $[9,10],(\rightarrow)$

$(\rightarrow)$ Voir la Nouvelle de S. Rousseaux et al., $\mathrm{m} / \mathrm{s} \mathrm{n}^{\circ} 2$, février 2010 , page 130

un événement qui

pourrait agir en favorisant l'échange des molécules $\mathrm{H} 2 \mathrm{~A}$ déjà présentes dans le nucléosome avec le variant H2A.L.2 néo-synthétisé, par un accroissement de la dynamique chromatinienne.

En conclusion, ces nouvelles données incitent à reconsidérer le scénario classique impliquant l'action successive des protéines de transition puis des protamines. En effet, l'incorporation de I'histone H2A.L.2 accélère la transformation des nucléosomes en induisant l'action presque simultanée des protéines de transition et des protamines. Ainsi, l'incorporation du variant d'histone H2A.L. 2 initie la transformation des nucléosomes et correspond à une nouvelle étape, antérieure au remplacement des histones (Figure 1). $\diamond$

The mystery of histone disappearance during spermatogenesis

\section{LIENS D'INTÉRÊT}

Les auteurs déclarent n'avoir aucun lien d'intérêt concernant les données publiées dans cet article.

\section{RÉFÉRENCES}

1. Gaucher J, Reynoird N, Montellier $\varepsilon$, et al. From meiosis to postmeiotic events: the secrets of histone disappearance. FEBS J $2010 ; 277$ : 599-604.

2. Lewis JD, Abbott DW, Ausio J. A haploid affair: core histone transitions during spermatogenesis. Biochem Cell Biol $2003 ; 81$ : 131-40.

3. Meistrich ML, Mohapatra B, Shirley CR, et al. Roles of transition nuclear proteins in spermiogenesis. Chromosoma 2003 ; 111 : 483-8.

4. Zhao M, Shirley CR, Mounsey S, et al. Nucleoprotein transitions during spermiogenesis in mice with transition nuclear protein Tnpl and Tnp2 mutations. Biol Reprod 2004 ; 71 : 1016-25.

5. Shirley CR, Hayashi S, Mounsey S, et al. Abnormalities and reduced reproductive potential of sperm from Tnpl-and Tnp2-null double mutant mice. Biol Reprod $2004 ; 71: 1220-9$.

6. Zhao M, Shirley CR, Hayashi S, et al. Transition nuclear proteins are required for normal chromatin condensation and functional sperm development. Genesis 2004 ; 38 : 200-13.

7. Govin J, Escoffier \&, Rousseaux S, et al. Pericentric heterochromatin reprogramming by new histone variants during mouse spermiogenesis. J Cell Biol $2007 ; 176: 283-94$.

8. Barral S, Morozumi Y, Tanaka H, et al. Histone variant H2A.L.2 guides transition protein-dependent protamine assembly in male germ cells. Mol Cell 2017 ; $66: 89-101$.

9. Goudarzi A, Shiota H, Rousseaux S, et al. Genomescale acetylation-dependent histone eviction during spermatogenesis. J Mol Biol 2014 ; 426 : 3342-9.

10. Rousseaux S, Petosa C, Müller CW, et al. Du nouveau dans la compréhension de la reprogrammation postméiotique du génome mâle. Med Sci (Paris) $2010 ; 26: 130-2$

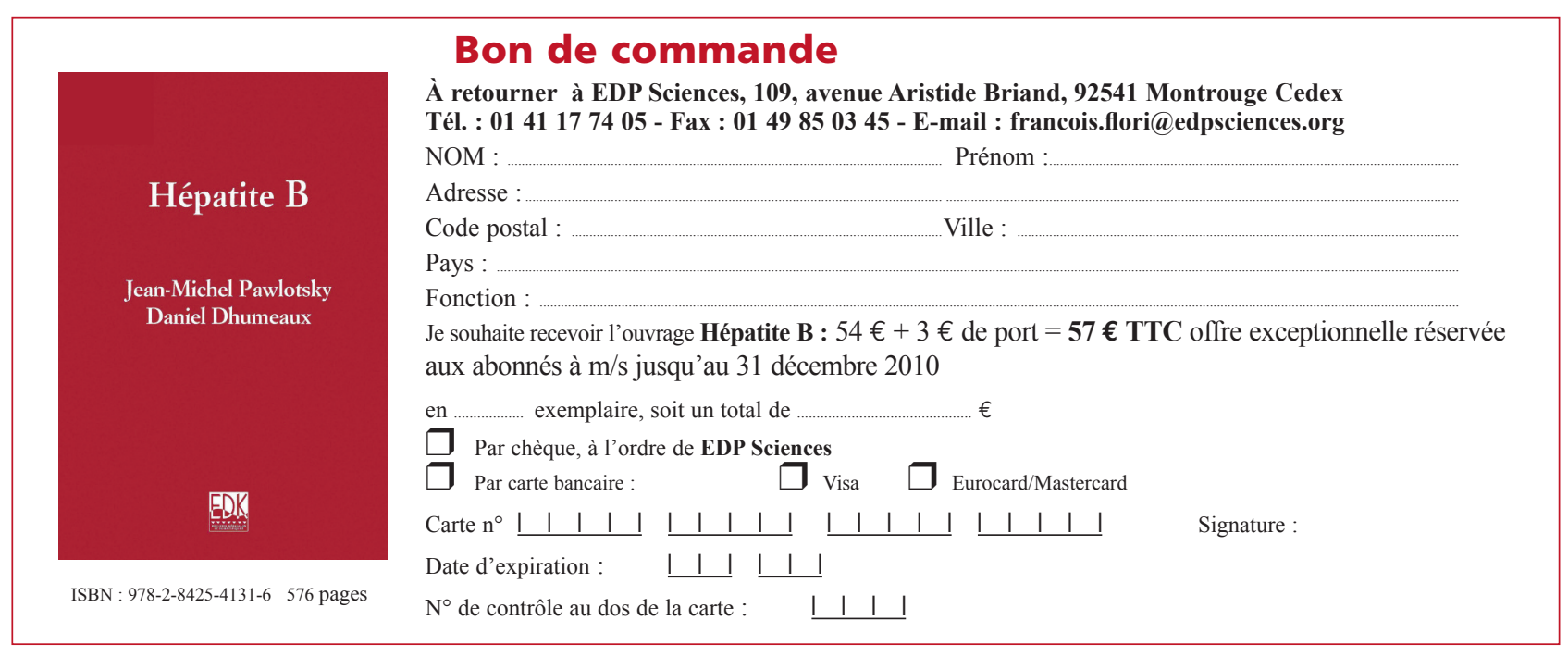

